2

\title{
Multilevel societies in birds
}

\author{
Danai Papageorgiou* ${ }^{1,2,3}$, Damien Farine* 1,2,3
}

1. Max Planck Institute of Animal Behavior, Department of Collective Behavior, Universitätsstraße 10, Konstanz, 78457, Germany

2. University of Konstanz, Department of Biology, Universitätsstraße 10, Konstanz, 78457, Germany

3. University of Konstanz, Center for the Advanced Study of Collective Behaviour, Universitätsstraße 10, Konstanz, 78457, Germany

Correspondence to: dpapageorgiou@,ab.mpg.de, dfarine@ab.mpg.de

Twitter accounts: @DanPapageorgiou,@DamienFarine

Author contributions: Both authors conceived the study and contributed equally to the writing.

Keywords: avian societies, complex sociality, social structure, social organisation 


\section{Letter}

There is growing interest in the study of multilevel societies, where social units comprising several individual animals (human and non-human) come together to form higher-level groupings. Grueter et al. [1] provide a useful definition of multilevel societies, highlighting that key characteristics of multilevel societies should include stability across different social levels and that stability should be driven by active social preferences rather than attraction to the same resource or spatial overlap. Despite their framework being predominately focused on mammals, and in particular primates, we argue it applies to a much wider-range of taxa. Here, we highlight the important contribution that studies on birds can make to distinguish different evolutionary pathways that can lead to multilevel societies and mechanisms that might maintain these.

Birds have a wide diversity of social systems, with many species living in nested societies. Studies on birds have a long history of conducting comparative studies within and across phylogenetic lineages [2]. It is now also abundantly clear that birds can possess cognitive abilities similar to mammals [3], and the means of communicating through group- and subgroup-level acoustic signatures [4], thereby highlighting that birds have the capacity to maintain multilevel societies. They therefore represent an ideal taxonomic group to help with defining and distinguishing multilevel societies from other forms of nested social structures. For example, species living in two-level societies exhibit different combinations of stability and cohesion at upper (e.g. groups or colonies) and lower levels (e.g. pairs or breeding units). Colonial cooperative-breeding sociable weavers (Philetairus socius) collectively build large nests and maintain long-term colony membership. Colony members forage together, with individuals expressing stronger cohesion with members of the same cooperative unit within foraging flocks [5]. In non-cooperative colonial breeders, such as zebra finches (Taeniopygia guttata), individuals maintain long-term stable pair bonds, and forage cohesively with other pairs with whom they breed synchronously, carrying-over these between-pair bonds into subsequent breeding seasons [6]. While colony membership in zebra finches is unstable within and across years, other species such as slender-billed gulls (Chroicocephalus genei) maintain long-term colony membership despite colonies relocating large distances between years [7], forming a stable upper level that suggests underlying social structure. Beyond colonial breeders, clans of thornbills (e.g. the buff-rumped thornbill Acanthiza reguloides) can be stable and consist of multiple cooperative-breeding groups, with clans foraging cohesively but splitting into smaller groups during breeding [8]. 
There is also emerging evidence that group-living birds can form three-level societies. Bell miners (Manorina melanophrys) live in large colonies that comprise of cooperative-breeding units (lower level) that are members of coteries (intermediate level) which are then part of a colony (upper level), although there is no evidence that the upper level is socially-driven and not arising simply from attraction to the same resource (the colony) [9]. By contrast, vulturine guineafowl (Acryllium vulturinum) move cohesively in groups that are stable across seasons (intermediate level), and groups roost and move preferentially with other groups (upper level), but the reproductive units within the group (lower level) can change from one season to the next [10]. Thus, stability is not necessarily equally distributed across levels, and birds may provide an ideal set of species to develop a deeper understanding of how cohesion and social stability define different types of multilevel societies (Fig. 1). Although Grueter et al. [1] defines the lower level as the core unit, this might misrepresent societies where membership in the society is clearest at intermediate (e.g. groups of vulturine guineafowl) or higher (e.g. colonies of slender-billed gulls) levels. Studies from birds can also allow social versus nonsocial drivers of nestedness to be disentangled. While multilevel societies can emerge from social preferences, seemingly identical patterns can arise from spatial and resource-driven processes. Simply studying patterns of social structure arising at larger ecological scales will undoubtedly uncover community sub-structuring driven by resource distribution and habitat configuration [11]. For example, songbirds can maintain consistent community structure, at two spatial scales, that is sufficiently stable to maintain experimentally-induced local traditions across generations [12]. However, such community structure arises through a combination of individual differences in micro-habitat preferences (lower level), and habitat geometry restricting the movement of individuals across the woodland (upper level) (see [11]). This example highlights how studies in birds can help reveal mechanisms that generate patterns of social structure that are consistent with those from multilevel societies, even to the point of exhibiting some of the same seemingly-adaptive 'behaviours' as multilevel societies (local traditions), but without any social preferences taking place at higher levels.

We hope that Grueter et al. [1]'s work will inspire research on multilevel societies in birds. Much can be gained by expanding existing evidence of complex and nested avian societies into the multilevel society framework. When implementing this framework, studies will need to explicitly consider the number of levels, their stability and cohesion, and the mechanisms underlying the emergence and/or maintenance of each level. In doing so, studies on birds will 
help with developing a better mechanistic understanding of multilevel societies, and whether, regardless of the drivers, individuals can reap benefits from living in a nested population structure, such as information transmission.

\section{References}

1 Grueter, C.C. et al. (2020) Multilevel Organisation of Animal Sociality. Trends Ecol. Evol 9, 834-847.

2 Riehl, C. (2013) Evolutionary routes to non-kin cooperative breeding in birds. Proc. R. Soc. B Biol. Sci. 280, 20132245.

3 Stacho, M. et al. (2020) A cortex-like canonical circuit in the avian forebrain. Science 369 , eabc5534.

4 Henry, L. et al. (2015) Dialects in Animals: Evidence, Development and Potential Functions. Anim. Behav. Cogn. 2, 132-155

5 Ferreira, A.C. et al. (2020) How to make methodological decisions when inferring social networks. Ecol. Evol. 17, 9132-9143.

Brandl, H.B. et al. (2019) Wild zebra finches that nest synchronously have long-term stable social ties. J. Anim. Ecol. 00: 1-11

7 Francesiaz, C. et al. (2017) Familiarity drives social philopatry in an obligate colonial breeder with weak interannual breeding-site fidelity. Anim. Behav. 124, 125-133

8 Bell, H.L. and Ford, H.A. (1986) A comparison of the social organization of three syntopic species of Australian thornbill, Acanthiza. Behav. Ecol. Sociobiol. 19, 381392

9 Painter, J.N. et al. (2000) Complex social organization reflects genetic structure and relatedness in the cooperatively breeding bell miner, Manorina melanophrys. Mol. Ecol. 9, 1339-1347

10 Papageorgiou, D. et al. (2019) The multilevel society of a small-brained bird. Curr. Biol. 29, R1120-R1121

11 He, P. et al. (2019) The role of habitat configuration in shaping social structure: a gap in studies of animal social complexity. Behav. Ecol. Sociobiol. 73, 9

12 Aplin, L.M. et al. (2015) Experimentally induced innovations lead to persistent culture via conformity in wild birds. Nature 518, 538-541 


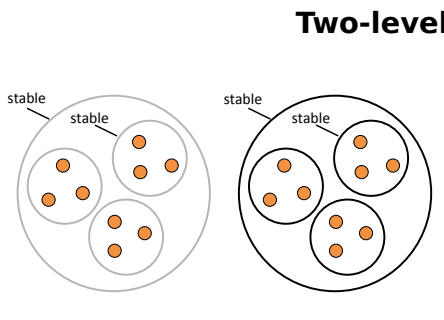

Great tits
Sociable weavers

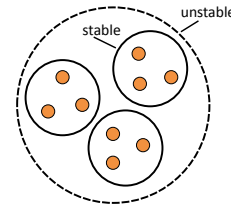
Buff-rumped thornbills
Zebra finches

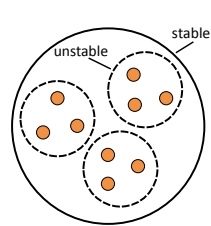

Slender-billed gulls

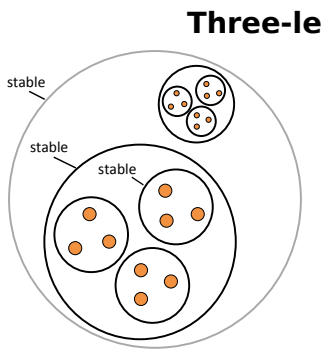

Bell miners

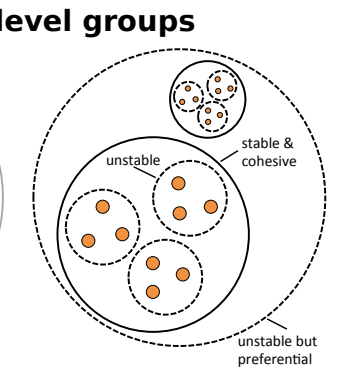

Vulturine guineafowls

134 Figure 1. The levels of nested societies can differ in their stability and cohesiveness, and non-

135 social drivers can operate at all levels. Great tits (Parus major) form fission-fusion flocks that are non-randomly drawn from the local population and that live within a repeatable community structure, but while flocks appear cohesive, their membership is highly dynamic and the social structure at both levels is driven by underlying habitat characteristics. In sociable weavers (Philetairus socius), individuals form stable breeding groups that maintain long-term membership to a colony, which they construct, and individuals forage cohesively with members of their cooperative unit and colony. Zebra finches (Taeniopygia guttata) form long-term stable pairs, but membership to the colony is unstable both within and between reproductive seasons, while slender-billed gulls (Chroicocephalus genei) maintain colony membership across years even when these relocate large distances, but little is known about their wintering social behaviour. Some species exhibit greater cohesion and stability outside of breeding. For example, buff-rumped thornbills (Acanthiza reguloides) winter in clans, but these break up into multiple cooperatively-breeding units during the reproductive season. In three-level societies, bell miners live (Manorina melanophrys) in large colonies, which consist of cooperatively-breeding units that form coteries, but there does not appear to be any social interactions among coteries within the same colony. Finally, vulturine guineafowl (Acryllium vulturinum) form stable and cohesive social groups that comprise of changing breeding pairs, and social groups preferentially interact with other social groups. Dashed lines represent lack of stability or social cohesion, and grey lines represent resource-driven levels. 